\title{
Third Hankel Determinant Problem for Certain Subclasses of Analytic Functions Associated with Nephroid Domain
}

\section{Muhammad Ghaffar Khan ${ }^{1}$, Bakhtiar Ahmad ${ }^{2}$, Wali Khan Mashwani ${ }^{1}$, Timilehin Gideon Shaba ${ }^{3, *}$ and Muhammad Arif ${ }^{4}$}

${ }^{1}$ Institute of Numerical Sciences, Kohat University of Science and Technology, Kohat, Pakistan e-mail: ghaffarkhan020@gmail.com, mashwanigr8@gmail.com

2 Govt. Degree College Mardan, 23200 Mardan, Pakistan

e-mail: pirbakhtiarbacha@gmail.com

${ }^{3}$ Department of Mathematics, University of Ilorin, P. M. B. 1515, Ilorin, Nigeria e-mail: shabatimilehin@gmail.com

${ }^{4}$ Department of Mathematics, Abdul Wali Khan University, 23200, Mardan, KP, Pakistan e-mail: marifmaths@awkum.edu.pk

\begin{abstract}
In this research article we consider two well known subclasses of starlike and bounded turning functions associated with nephroid domain. Our aims to find third Hankel determinant for these classes.
\end{abstract}

\section{Introduction and Definitions}

Let $\mathcal{A}$ be the collections of all normalized analytic functions defined in the unit $\operatorname{disc} \mathfrak{D}=\{z \in \mathbb{C}:|z|<1\}$ and of the form

$$
f(z)=z+\sum_{n=2}^{\infty} a_{n} z^{n}, \quad(z \in \mathfrak{D}) .
$$

Received: February 15, 2021; Accepted: March 24, 2021

2010 Mathematics Subject Classification: 30C45, 30D30.

Keywords and phrases: analytic functions, sine hyperbolic function, subordination.

${ }^{*}$ Corresponding author 
Let us denote the most basic, well known and important subclass of class $\mathcal{A}$ by $\mathcal{S}$ which consists of all univalent functions in $\mathfrak{D}$. Since in the early stage of 20 th century, researchers have been interested in coefficients of function $f$ in class $\mathcal{A}$. In year 1916, Bieberbach see [1] was first to discover a coefficient conjecture for the function $f \in \mathcal{S}$ and finally in year 1985 De-Branges [2] solved Bieberbach coefficient conjecture. In era 1916 to 1985 many researcher have tried to prove or disprove this conjecture and they discovered different subclasses of the class $\mathcal{S}$ associated with different image domains. The definition of class $\mathcal{S}^{*}, \mathcal{C}$ and $\mathcal{R}$ which are subclasses of class $\mathcal{S}$ can be written in terms of subordination

$$
\begin{aligned}
\mathcal{S}^{*} & =\left\{f \in \mathcal{S}: \frac{z f^{\prime}(z)}{f(z)} \prec \frac{1+z}{1-z}, \quad z \in \mathfrak{D}\right\}, \\
\mathcal{C} & =\left\{f \in \mathcal{S}: \frac{\left(z f^{\prime}(z)\right)^{\prime}}{f^{\prime}(z)} \prec \frac{1+z}{1-z}, \quad z \in \mathfrak{D}\right\}, \\
\mathcal{R} & =\left\{f \in \mathcal{S}: f^{\prime}(z) \prec \frac{1+z}{1-z}, \quad z \in \mathfrak{D}\right\},
\end{aligned}
$$

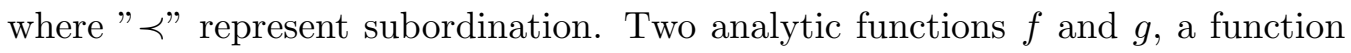
$f$ is subordinate to $g$ symbolically $f \prec g$ if there exist an analytic function $w(z)$ with limitation $w(0)=0$ and $|w(z)|<|z|$ such that $f(z)=g(w(z))$. If $g \in \mathcal{S}$, then equivalence conditions

$$
f(0)=g(0) \text { and } f(\mathfrak{D}) \subset g(\mathfrak{D}) .
$$

Let $\mathcal{P}$ denote class of all analytic functions $p$ such that $\operatorname{Re}(p(z))>0$, and of the form

$$
p(z)=z+\sum_{n=1}^{\infty} c_{n} z^{n}, \quad(z \in \mathfrak{D}) .
$$

By changing the function right hand side of subordinations in 1.2, we obtain some subclasses of the class $\mathcal{S}$ which have interesting geometric properties, see [3, 4, 5, 6, 7, 8, 9, 10, 11, 12, 13. From among these subfamilies we recall here 
the families that are associated with trigonometric function as follows;

$$
\begin{aligned}
& \mathcal{S}_{\mathcal{N} e}^{*}=\left\{f \in \mathcal{S}: \frac{z f^{\prime}(z)}{f(z)} \prec 1+z-\frac{1}{3} z^{3}\right\}, \quad(z \in \mathfrak{D}), \\
& \mathcal{S}_{\mathcal{N} e}^{*}=\left\{f \in \mathcal{S}: f^{\prime}(z) \prec 1+z-\frac{1}{3} z^{3}\right\}, \quad(z \in \mathfrak{D}) .
\end{aligned}
$$

Recently, authors in [18, introduced the class $\mathcal{S}_{\mathcal{N} e}^{*}$ which are associated with nephriod domain.

The Hankel determinant $H_{q, n}(f)$ where parameters $q, n \in \mathbb{N}=\{1,2,3, \cdots\}$ for function $f \in \mathcal{S}$ of the form (1.1) was introduced by Pommerenke [14, 15] as;

$$
H_{q, n}(f)=\left|\begin{array}{llll}
a_{n} & a_{n+1} & \ldots & a_{n+q-1} \\
a_{n+1} & a_{n+2} & \ldots & a_{n+q} \\
\vdots & \vdots & \ldots & \vdots \\
a_{n+q-1} & a_{n+q} & \ldots & a_{n+2 q-2}
\end{array}\right| .
$$

The growth of $H_{q, n}(f)$ has been evaluated for different subcollections of univalent functions. Exceptionally, the sharp bound of the determinant $H_{2,2}(f)=$ $\left|a_{2} a_{4}-a_{3}^{2}\right|$ for class $\mathcal{S}^{*}, \mathcal{C}$ and $\mathcal{R}$ were found by Janteng et al. [16, 17] while for the family of close-to-convex functions the sharp estimation is still unknown (see, [19]). On the other hand, for the set of Bazilevič functions, the best estimate of $\left|H_{2,2}(f)\right|$ was proved by Krishna et al. [20]. For more work on $H_{2,2}(f)$, see [21, 22, 23, 24, 25].

$$
H_{3,1}(f)=\left|\begin{array}{ccc}
1 & a_{2} & a_{3} \\
a_{2} & a_{3} & a_{4} \\
a_{3} & a_{4} & a_{5}
\end{array}\right|
$$

is known as third order Hankel determinant and the estimation of this determinant $\left|H_{3,1}(f)\right|$ is so hard. In 2010, the first article on $H_{3,1}(f)$ by Babalola [26], in which he obtained the upper bound of $\left|H_{3,1}(f)\right|$ for the groups of $\mathcal{S}^{*}, \mathcal{K}$ and $\mathcal{R}$. Later on, a few creators distributed their work regarding $\left|H_{3,1}(f)\right|$ for various subcollections of holomorphic and univalent functions, see [34, 35, 36, 37, 38, which served as a base for the research in this field. Recently various authors explored some interesting classes for the said property of Hankel determinant. 
Srivastava et al. 27] discussed this result for a class of Bi-valent functions defined by $q$-derivative and gave various interesting properties of it. Then he along with coauthors in [28] investigated the class of close to convex functions associated with lemniscate of Bernouli and evaluated its Hankel determinant. Continuing the same trend he in [29] incorporated the research on Toeplitz forms and Hankel determinant for some $q$-starlike functions associated with a generalized domain. Many other domains were also investigated for its Hankel determinant like a class of starlike functions associated with $k$-Fibonacci numbers. Whose third Hankel was evaluated by Shafiq et al. [30]. Further related work on the subject the reader is referred to [31, 32, 33]. Motivated from above discussed work on the topic we investigate $\left|H_{3,1}(f)\right|$ for classes of functions defined in the relations (1.4) and 1.5$)$.

\section{Sets of Lemma}

The following lemmas are important as they help in our main results.

Lemma 1. If $p(z) \in \mathcal{P}$ and it is of the form 1.3 , then

$$
\begin{aligned}
\left|c_{n}\right| & \leq 2 \text { for } n \geq 1, \\
\left|c_{n+k}-\delta c_{n} c_{k}\right| & \leq \begin{cases}2 & \text { for } 0 \leq \delta \leq 1, \\
2|2 \delta-1| & \text { elsewhere. }\end{cases} \\
\left|c_{n} c_{m}-c_{l} c_{k}\right| & \leq 4 \text { for } n+m=l+k, \\
\left|c_{n+2 k}-\delta c_{n} c_{k}^{2}\right| & \leq 2(1+2 \delta) \text { for } \delta \in \mathbb{R}, \\
\left|c_{2}-\frac{c_{1}^{2}}{2}\right| & \leq 2-\frac{\left|c_{1}\right|^{2}}{2},
\end{aligned}
$$

and for $\xi \in \mathbb{C}$

$$
\left|c_{2}-\xi c_{1}^{2}\right| \leq 2 \max \{1 ;|2 \xi-1|\} .
$$

For the results in 2.1], 2.2, 2.3), 2.4, 2.5 see 44. Also see 43. for 2.6. 
Lemma 2. 46]. If $p(z) \in \mathcal{P}$ and is represented by [1.3), then

$$
\left|c_{2}-\nu c_{1}^{2}\right| \leq\left\{\begin{array}{lc}
-4 \nu+2 & (\nu \leq 0) \\
2 & (0 \leq \nu \leq 1) \\
4 \nu-2 & (\nu \geq 1)
\end{array}\right.
$$

Lemma 3. 47]. If $p(z) \in \mathcal{P}$ and is represented by [1.3), then

$$
\left|a c_{1}^{3}-b c_{1} c_{2}+d c_{3}\right| \leq 2|a|+2|b-2 a|+2|a-b+d| .
$$

\section{Bounds of $\left|H_{3,1}(f)\right|$ for class $\mathcal{S}_{\mathcal{N} e}^{*}$}

Theorem 1. Let $f \in \mathcal{S}_{\mathcal{N} e}^{*}$ of the form 1.1. Then

$$
\begin{aligned}
& \left|a_{2}\right| \leq 1 \\
& \left|a_{3}\right| \leq \frac{1}{2} \\
& \left|a_{4}\right| \leq \frac{7}{18} \\
& \left|a_{5}\right| \leq \frac{5}{12} .
\end{aligned}
$$

The first two bounds are sharp.

Proof. Since $f \in \mathcal{S}_{\mathcal{N} e}^{*}$, there exists an analytic function $s(z),|s(z)| \leq 1$ and $s(0)=0$, such that

$$
\frac{z f^{\prime}(z)}{f(z)}=1+w(z)-\frac{1}{3}(w(z))^{3} .
$$

Denote

$$
\Psi(s(z))=1+w(z)-\frac{1}{3}(w(z))^{3},
$$

and

$$
k(z)=1+c_{1} z+c_{2} z^{2}+\cdots=\frac{1+w(z)}{1-w(z)} .
$$

Obviously, the function $k(z) \in \mathcal{P}$, and $w(z)=\frac{k(z)-1}{k(z)+1}$. This gives

$$
w(z)=\frac{k(z)-1}{k(z)+1}=\frac{c_{1} z+c_{2} z^{2}+c_{3} z^{3}+\cdots}{2+c_{1} z+c_{2} z^{2}+c_{3} z^{3}+\cdots} .
$$


And

$$
\begin{aligned}
& 1+(w(z))-\frac{1}{3}(w(z))^{3} \\
= & 1+\frac{1}{2} c_{1} z+\left(\frac{1}{2} c_{2}-\frac{1}{4} c_{1}^{2}\right) z^{2}+\left(\frac{1}{12} c_{1}^{3}-\frac{1}{2} c_{2} c_{1}+\frac{1}{2} c_{3}\right) z^{3} \\
& +\left(\frac{1}{4} c_{1}^{2} c_{2}-\frac{1}{2} c_{3} c_{1}-\frac{1}{4} c_{2}^{2}+\frac{1}{2} c_{4}\right) z^{4}+\cdots .
\end{aligned}
$$

And other side,

$$
\begin{aligned}
\frac{z f^{\prime}(z)}{f(z)}= & 1+a_{2} z+\left(2 a_{3}-a_{2}^{2}\right) z^{2}+\left(a_{2}^{3}-3 a_{2} a_{3}+3 a_{4}\right) z^{3}+ \\
& \left(-a_{2}^{4}+4 a_{2}^{2} a_{3}-4 a_{2} a_{4}-2 a_{3}^{2}+4 a_{5}\right) z^{4}+\cdots .
\end{aligned}
$$

On equating coefficients of (3.3), and (3.4), we get

$$
\begin{aligned}
& a_{2}=\frac{1}{2} c_{1}, \\
& a_{3}=\frac{1}{4} c_{2}, \\
& a_{4}=-\frac{1}{72} c_{1}^{3}-\frac{1}{24} c_{2} c_{1}+\frac{1}{6} c_{3}, \\
& a_{5}=\frac{5}{576} c_{1}^{4}-\frac{1}{48} c_{1}^{2} c_{2}-\frac{1}{24} c_{3} c_{1}-\frac{1}{32} c_{2}^{2}+\frac{1}{8} c_{4} .
\end{aligned}
$$

Now using (2.1) in (3.5) and 3.6), we get

$$
\left|a_{2}\right| \leq 1 \text { and }\left|a_{3}\right| \leq \frac{1}{2} .
$$

Rearrange the equation (3.7), we may write

$$
\left|a_{4}\right|=\left|\frac{1}{12}\left(c_{3}-\frac{1}{2} c_{1} c_{2}\right)+\frac{1}{12}\left(c_{3}-\frac{1}{6} c_{1}^{3}\right)\right| .
$$

Using triangle inequality along with $(2.2)$ and $(2.4)$, we get

$$
\left|a_{4}\right| \leq \frac{7}{18} \text {. }
$$

Now rearrange the 3.8 , we may write

$$
\left|a_{5}\right|=\left|\frac{1}{16}\left(c_{4}-\frac{2}{3} c_{1} c_{3}\right)+\frac{1}{16}\left(c_{4}-\frac{1}{2} c_{2}^{2}\right)-\frac{c_{1}^{2}}{48}\left(c_{2}-\frac{5}{12} c_{1}^{2}\right)\right|
$$


Application of triangle inequality along with 2.1) and (2.2), we get

$$
\left|a_{5}\right| \leq \frac{5}{12}
$$

The first two bounds are sharp for function define as

$$
f(z)=z \exp \left(z-\frac{z^{3}}{9}\right)=z+z^{2}+\frac{z^{3}}{2}+\cdots .
$$

Theorem 2. Let $f(z) \in \mathcal{S}_{\mathcal{N} e}^{*}$ be of the form 1.1. Then

$$
\left|a_{3}-\lambda a_{2}^{2}\right| \leq\left\{\begin{array}{cc}
\frac{1-2 \lambda}{2}, & \lambda \leq 0 \\
\frac{1}{2}, & 0 \leq \lambda \leq 1 \\
\frac{2 \lambda-1}{2}, & \lambda \geq 1 .
\end{array} .\right.
$$

Proof. Since using (3.5) and (3.6), we get

$$
\left|a_{3}-\lambda a_{2}^{2}\right|=\frac{1}{4}\left|c_{2}-\lambda c_{1}^{2}\right|
$$

applying Lemma 2, we get the required results.

Theorem 3. Let $f(z) \in \mathcal{S}_{\mathcal{N} e}^{*}$ be of the form 1.1. Then for $\xi \in \mathbb{C}$, we have

$$
\left|a_{3}-\xi a_{2}^{2}\right| \leq \frac{1}{2} \max \{1,|2 \xi-1|\} .
$$

Proof. Since using (3.5) and (3.6), we get

$$
\left|a_{3}-\xi a_{2}^{2}\right|=\frac{1}{4}\left|c_{2}-\xi c_{1}^{2}\right|,
$$

applying Lemma 2.6, we get the required results.

If we put $\xi=1$, the above result become as:

Theorem 4. Let $f(z) \in \mathcal{S}_{\mathcal{N} e}^{*}$ be of the form 11.1. Then

$$
\left|a_{3}-a_{2}^{2}\right| \leq \frac{1}{2}
$$

This results is sharp. 
Theorem 5. Let $f(z) \in \mathcal{S}_{\mathcal{N} \text { e }}^{*}$ be of the form 11.1 . Then

$$
\left|a_{2} a_{3}-a_{4}\right| \leq \frac{7}{18}
$$

Proof. Since using (3.5), (3.6) and (3.7) also rearranging term, we get

$$
\left|a_{2} a_{3}-a_{4}\right|=\left|\frac{1}{12}\left(c_{3}-c_{2} c_{1}\right)+\frac{1}{12}\left(c_{3}-\frac{1}{6} c_{1}^{3}\right)\right|,
$$

Using triangle inequality along with 2.2 and $(2.4)$, we get

$$
\left|a_{2} a_{3}-a_{4}\right| \leq \frac{7}{18}
$$

Theorem 6. Let $f(z) \in \mathcal{S}_{\mathcal{N} e}^{*}$ be of the form 1.1. Then

$$
\left|a_{2} a_{4}-a_{3}^{2}\right| \leq \frac{4}{9}
$$

Proof. Since using (3.5), 3.6) and (3.7), we get

$$
\begin{aligned}
\left|a_{2} a_{4}-a_{3}^{2}\right| & =\left|-\frac{1}{144} c_{1}^{4}-\frac{1}{48} c_{1}^{2} c_{2}+\frac{1}{12} c_{3} c_{1}-\frac{1}{16} c_{2}^{2}\right| \\
& =\left|\frac{1}{16}\left(c_{1} c_{3}-c_{2}^{2}\right)+\frac{1}{48} c_{1}\left(c_{3}-c_{1} c_{2}\right)-\frac{1}{144} c_{1}^{4}\right|,
\end{aligned}
$$

applying 2.1), 2.2 and 2.3), we get the required result.

Theorem 7. Let $f(z) \in \mathcal{S}_{\mathcal{N} \text { e }}^{*}$ be of the form 11.1 . Then

$$
\left|H_{3,1}(f)\right| \leq \frac{377}{648} \simeq 0.58179 \text {. }
$$

Proof. Since

$$
H_{3,1}(f)=a_{3}\left(a_{2} a_{4}-a_{3}^{2}\right)-a_{4}\left(a_{4}-a_{2} a_{3}\right)+a_{5}\left(a_{3}-a_{2}^{2}\right),
$$

by applying triangle inequality, we obtain

$$
\left|H_{3,1}(f)\right| \leq\left|a_{3}\right|\left|a_{2} a_{4}-a_{3}^{2}\right|+\left|a_{4}\right|\left|a_{4}-a_{2} a_{3}\right|+\left|a_{5}\right|\left|a_{3}-a_{2}^{2}\right| .
$$

Next, from Theorems 1, 4, 5and 6, we get the required result. 


\section{Bounds of $\left|H_{3,1}(f)\right|$ for class $\mathcal{R}_{\mathcal{N} e}$}

Theorem 8. Let $f \in \mathcal{R}_{\mathcal{N} e}$ of the form 1.1 . Then

$$
\begin{aligned}
& \left|a_{2}\right| \leq \frac{1}{2}, \\
& \left|a_{3}\right| \leq \frac{1}{3}, \\
& \left|a_{4}\right| \leq \frac{1}{4}, \\
& \left|a_{5}\right| \leq \frac{3}{5} .
\end{aligned}
$$

Proof. Since $f \in \mathcal{R}_{\mathcal{N} e}$, there exists an analytic function $w(z),|w(z)| \leq 1$ and $w(0)=0$, such that

$$
f^{\prime}(z)=1+w(z)-\frac{1}{3}(w(z))^{3}
$$

And

$$
f^{\prime}(z)=1+2 a_{2} z+3 a_{3} z^{2}+3 a_{4} z^{3}+4 a_{5} z^{4}+\cdots .
$$

On equating coefficients of (3.3), and (4.1), we get $\left(\frac{1}{6} c_{2}-\frac{1}{12} c_{1}^{2}\right)^{2}=\frac{1}{144} c_{1}^{4}-\frac{1}{36} c_{1}^{2} c_{2}+$ $\frac{1}{36} c_{2}^{2}$

$$
\begin{aligned}
a_{2} & =\frac{1}{4} c_{1}, \\
a_{3} & =\frac{1}{6} c_{2}-\frac{1}{12} c_{1}^{2}, \\
a_{4} & =\frac{1}{48} c_{1}^{3}-\frac{1}{8} c_{2} c_{1}+\frac{1}{8} c_{3}, \\
a_{5} & =\frac{1}{20} c_{1}^{2} c_{2}-\frac{1}{10} c_{3} c_{1}-\frac{1}{20} c_{2}^{2}+\frac{1}{10} c_{4} .
\end{aligned}
$$

Now using 2.1 to 4.2, we get

$$
\left|a_{2}\right| \leq \frac{1}{2}
$$

Using (2.5) to (4.3), we obtain

$$
\left|a_{3}\right| \leq \frac{1}{3}
$$


Application of Lemma 3 to 4.4 , lead us to

$$
\left|a_{4}\right| \leq \frac{1}{4}
$$

Rearranging the 4.5, we have

$$
\left|a_{5}\right|=\left|\frac{1}{10}\left(c_{4}-\frac{1}{2} c_{2}^{2}\right)-\frac{c_{1}}{10}\left(c_{3}-\frac{1}{2} c_{1} c_{2}\right)\right|,
$$

applying 2.1) and 2.2), we get

$$
\left|a_{5}\right| \leq \frac{3}{5}
$$

Theorem 9. Let $f(z) \in \mathcal{R}_{\mathcal{N} e}$ be of the form 1.1). Then for $\xi \in \mathbb{C}$, we have

$$
\left|a_{3}-\xi a_{2}^{2}\right| \leq \frac{1}{3} \max \left\{1, \frac{3|\xi|}{4}\right\} .
$$

Proof. Since using (4.2) and (4.3), we get

$$
\left|a_{3}-\xi a_{2}^{2}\right|=\frac{1}{6}\left|c_{2}-\frac{4+3 \xi}{8} c_{1}^{2}\right|,
$$

application of relation (2.6), we get the required results.

If we put $\xi=1$, the above result become as:

Theorem 10. Let $f(z) \in \mathcal{R}_{\mathcal{N} \text { e }}$ be of the form 1.1). Then

$$
\left|a_{3}-a_{2}^{2}\right| \leq \frac{1}{3}
$$

Theorem 11. Let $f(z) \in \mathcal{R}_{\mathcal{N} e}$ be of the form 1.1. Then

$$
\left|a_{2} a_{3}-a_{4}\right| \leq \frac{1}{4}
$$

Proof. Since using (4.2), 4.3) and 4.4, we get

$$
\left|a_{2} a_{3}-a_{4}\right|=\left|\frac{1}{24} c_{1}^{3}-\frac{1}{6} c_{2} c_{1}+\frac{1}{8} c_{3}\right|,
$$

using Lemma 3, we have

$$
\left|a_{2} a_{3}-a_{4}\right| \leq \frac{1}{4}
$$


Theorem 12. Let $f(z) \in \mathcal{R}_{\mathcal{N} e}$ be of the form (1.1). Then

$$
\left|a_{2} a_{4}-a_{3}^{2}\right| \leq \frac{11}{72}
$$

Proof. Since using (4.2), 4.3) and 4.4, we get

$$
\begin{aligned}
\left|a_{2} a_{4}-a_{3}^{2}\right| & =\left|-\frac{1}{576} c_{1}^{4}-\frac{1}{288} c_{1}^{2} c_{2}+\frac{1}{32} c_{3} c_{1}-\frac{1}{36} c_{2}^{2}\right| \\
& =\left|\frac{1}{36}\left(c_{1} c_{3}-c_{2}^{2}\right)+\frac{1}{288} c_{1}\left(c_{3}-c_{1} c_{2}\right)-\frac{1}{576} c_{1}^{4}\right|,
\end{aligned}
$$

application of triangle inequality along with 2.1), 2.2 and 2.3), we get the required result.

Theorem 13. Let $f(z) \in \mathcal{R}_{\mathcal{N} e}$ be of the form (1.1). Then

$$
\left|H_{3,1}(f)\right| \leq \frac{677}{2160} \simeq 0.31343 .
$$

Proof. Since

$$
H_{3,1}(f)=a_{3}\left(a_{2} a_{4}-a_{3}^{2}\right)-a_{4}\left(a_{4}-a_{2} a_{3}\right)+a_{5}\left(a_{3}-a_{2}^{2}\right)
$$

by applying triangle inequality, we obtain

$$
\left|H_{3,1}(f)\right| \leq\left|a_{3}\right|\left|a_{2} a_{4}-a_{3}^{2}\right|+\left|a_{4}\right|\left|a_{4}-a_{2} a_{3}\right|+\left|a_{5}\right|\left|a_{3}-a_{2}^{2}\right| .
$$

Next, from Theorems 8, 10, 11 and 12, we get the required result.

\section{Authors contributions}

All authors jointly worked on the results and they read and approved the final manuscript.

\section{Conflict of Interest}

The authors declare that there is no conflict of interests. 


\section{References}

[1] L. Bieberbach, Uber die koeffizienten derjenigen Potenzreihen, weiche eine schlichte Abbildung des Einheitskreises vermittein, Sitzungsberichte Preussische Akademie der Wissenschaften 138 (1916), 940-955.

[2] L. De Branges, A proof of the Bieberbach conjecture, Acta Math. 154 (1985), 137-152. https://doi.org/10.1007/BF02392821

[3] W. Janowski, Extremal problems for a family of functions with positive real part and for some related families, Ann. Polon. Math. 23 (1970), 159-177.

https://doi.org/10.4064/ap-23-2-159-177

[4] J. Sokół and J. Stankiewicz, Radius of convexity of some subclasses of strongly starlike functions, Zeszyty Nauk. Politech. Rzeszowskiej Mat. 19 (1996), 101-105.

[5] R.K. Raina and J. Sokół, Some properties related to a certain class of starlike functions, C. R. Math. Acad. Sci. Paris 353(11) (2015), 973-978. https://doi.org/10.1016/j.crma.2015.09.011

[6] R. Mendiratta, S. Nagpal and V. Ravichandran, On a subclass of strongly starlike functions associated with exponential function, Bull. Malays. Math. Sci. Soc. 38 (2015), 365-386. https://doi.org/10.1007/s40840-014-0026-8

[7] S. Kumar and V. Ravichandran, A subclass of starlike functions associated with a rational function, Southeast Asian Bull. Math. 40 (2016), 199-212.

[8] N.E. Cho, V. Kumar, S.S. Kumar and V. Ravichandran, Radius problems for starlike functions associated with the Sine function, Bull. Iran. Math. Soc. 45 (2019), 213-232. https://doi.org/10.1007/s41980-018-0127-5

[9] R. Kargar, A. Ebadian and J. Sokół, Radius problems for some subclasses of analytic functions, Complex Anal. Oper. Theory 11 (2017), 1639-1649.

https://doi.org/10.1007/s11785-016-0584-x

[10] R. Kargar, A. Ebadian and J. Sokół, On Booth lemniscate and starlike functions, Anal. Math. Phys. 9 (2019), 143-154.

https://doi.org/10.1007/s13324-017-0187-3 
[11] J. Dziok, R.K. Raina and J. Sokół, On a class of starlike functions related to a shell-like curve connected with Fibonacci numbers, Math. Comput. Model. 57 (2013), 1203-1211. https://doi.org/10.1016/j.mcm.2012.10.023

[12] N.E. Cho, S. Kumar, V. Kumar, V. Ravichandran and H.M. Srivastava, Starlike functions related to the Bell numbers, Symmetry 11 (2019), 219.

https://doi.org/10.3390/sym11020219

[13] S. Mahmood, H.M. Srivastava and S.N. Malik, Some subclasses of uniformly univalent functions with respect to symmetric points, Symmetry 11 (2019), 287. https://doi.org/10.3390/sym11020287

[14] Ch. Pommerenke, On the coefficients and Hankel determinants of univalent functions, J. Lond. Math. Soc. 41 (1966), 111-122.

https://doi.org/10.1112/jlms/s1-41.1.111

[15] Ch. Pommerenke, On the Hankel determinants of univalent functions, Mathematika 14 (1967), 108-112. https://doi.org/10.1112/S002557930000807X

[16] A. Jangteng, S.A. Halim and M. Darus, Coefficient inequality for a function whose derivative has a positive real part, J. Ineq. Pure Appl. Math. 7 (2006), 1-5.

[17] A. Jangteng, S.A. Halim and M. Darus, Coefficient inequality for starlike and convex functions, Int. J. Ineq. Math. Anal. 1 (2007), 619-625.

[18] L.A. Wani and A. Swaminathan, Stralike and convex functions associated with a Nephroid domain having cusps on the real axis, 2019. arXiv.1912.05767v1

[19] D. Răducanu and P. Zaprawa, Second Hankel determinant for close-to-convex functions, C. R. Math. Acad. Sci. Paris 355(10) (2017), 1063-1071. https://doi.org/10.1016/j.crma.2017.09.006

[20] D. V. Krishna and T. RamReddy, Second Hankel determinant for the class of Bazilevic functions, Stud. Univ. Babes-Bolyai Math. 60 (2015), 413-420.

[21] D. Bansal, Upper bound of second Hankel determinant for a new class of analytic functions, Appl. Math. Lett. 23 (2013), 103-107.

https://doi.org/10.1016/j.aml.2012.04.002 
[22] S.K. Lee, V. Ravichandran and S. Supramaniam, Bounds for the second Hankel determinant of certain univalent functions, J. Inequal. Appl. 2013, 2013:281, 17 pp. https://doi.org/10.1186/1029-242X-2013-281

[23] M. S. Liu, J. F. Xu and M. Yang, Upper bound of second Hankel determinant for certain subclasses of analytic functions, Abstr. Appl Anal. 2014, Art. ID 603180, 10 pp. https://doi.org/10.1155/2014/603180

[24] J. W. Noonan and D. K. Thomas, On the second Hankel determinant of areally mean $p$-valent functions, Trans. Amer. Math. Soc. 223 (1976), 337-346. https://doi.org/10.2307/1997533

[25] H. Orhan, N. Magesh and J. Yamini, Bounds for the second Hankel determinant of certain bi-univalent functions, Turk. J. Math. 40 (2016), 679-687. https://doi.org/10.3906/mat-1505-3

[26] K. O. Babalola, On $H_{3}(1)$ Hankel determinant for some classes of univalent functions, Inequal. Theory Appl. 6 (2010), 1-7.

[27] H. M. Srivastava, S. Altinkaya and S. Yalçin, Hankel determinant for a subclass of bi-valent functions defined by using a symmetric $q$-derivative operator, Filomat 32 (2018), 503-516. https://doi.org/10.2298/FIL1802503S

[28] H. M. Srivastava, Q. Z. Ahmad, M. Darus, N. Khan, B. Khan, N. Zaman and H. H. Shah, Upper bound of the third Hankel determinant for a subclass of close-to-convex functions associated with Lemniscate of Bernouli, Mathematics 7(9) (2019), 848. https://doi.org/10.3390/math7090848

[29] H. M. Srivastava, Q. Z. Ahmad, N. Khan, N. Khan and B. Khan, Hankel and Toeplitz determinant for a subclass of $q$-starlike functions associated with a general conic domain, Mathematics 7(2) (2019), 181. https://doi.org/10.3390/math7020181

[30] M. Shafiq, H. M. Srivastava, N. Khan, Q. Z. Ahmad, M. Darus and S. Kiran, An upper bound of the third Hankel determinant for a subclass of $q$-starlike functions associated with $k$-Fibonacci numbers, Symmetry 12(6) (2020), 1043. https://doi.org/10.3390/sym12061043

[31] L. Shi, M. G. Khan and B. Ahmad, Some geometric properties of a family of analytic functions involving a generalized q-operator, Symmetry 12(2) (2020), 291. https://doi.org/10.3390/sym12020291 
[32] M.G. Khan, B. Ahmad, G. Muraugusundaramoorthy, R. Chinram and W. K. Mashwani, Applications of modified sigmoid functions to a class of starlike functions, J. Funct. Spaces 2020, Art. ID 8844814, 8 pp. https://doi.org/10.1155/2020/8844814

[33] M.G. Khan, B. Ahmad, J. Sokol and Z. Muhammad, Coefficient problems in a class of functions with bounded turning associated with Sine function, European Journal of Pure and Applied Mathematics 14 (2021), 53-64. https://doi.org/10.29020/nybg.ejpam.v14i1.3902

[34] S. Altinkaya and S. Yalçin, Third Hankel determinant for Bazilevič functions, Advances in Math. 5 (2016), 91-96.

[35] D. Bansal, S. Maharana and J. K. Prajapat, Third order Hankel determinant for certain univalent functions, J. Korean Math. Soc. 52 (2015), 1139-1148. https://doi.org/10.4134/JKMS.2015.52.6.1139

[36] N.E. Cho, B. Kowalczyk, O.S. Kwon, A. Lecko and J. Sim, Some coefficient inequalities related to the Hankel determinant for strongly starlike functions of order alpha, J. Math. Inequal. 11 (2017), 429-439. https://doi.org/10.7153/jmi-2017-11-36

[37] D.V. Krishna, B. Venkateswarlua and T. RamReddy, Third Hankel determinant for bounded turning functions of order alpha, J. Niger. Math. Soc. 34 (2015), 121-127. https://doi.org/10.1016/j.jnnms.2015.03.001

[38] G. Shanmugam, B.A. Stephen and K.O. Babalola, Third Hankel determinant for $\alpha$-starlike functions, Gulf J. Math. 2 (2014), 107-113.

[39] P. Zaprawa, Third Hankel determinants for subclasses of univalent functions, Mediterr. J. Math. 14(1) (2017), Paper No. 19, 10 pp. https://doi.org/10.1007/s00009-016-0829-y

[40] B. Kowalczyk, A. Lecko and Y.J. Sim, The sharp bound of the Hankel determinant of the third kind for convex functions, Bull. Australian Math. Soc. 97 (2018), 435-445. https://doi.org/10.1017/S0004972717001125

[41] A. Lecko, Y.J. Sim and B. Śmiarowska, The sharp bound of the Hankel determinant of the third kind for starlike functions of order 1/2, Complex Anal. Oper. Theory 13(5) (2019), 2231-2238. https://doi.org/10.1007/s11785-018-0819-0 
[42] O.S. Kwon, A. Lecko and Y.J. Sim, The bound of the Hankel determinant of the third kind for starlike functions, Bull. Malays. Math. Sci. Soc. 42 (2018), 1-14. https://doi.org/10.1007/s40840-018-0683-0

[43] C. Pommerenke, Univalent Functions, Math. Lehrbucher, Band XXV, Vandenhoeck Ruprecht, Gottingen, 1975.

[44] F. Keogh and E. Merkes, A coefficient inequality for certain subclasses of analytic functions, Proc. Amer. Math. Soc. 20 (1969), 8-12. https://doi.org/10.1090/S0002-9939-1969-0232926-9

[45] V. Ravichandran and S. Verma, Bound for the fifth coefficient of certain starlike functions, C. R. Math. Acad. Sci. Paris 353(6) (2015), 505-510. https://doi.org/10.1016/j.crma.2015.03.003

[46] W.C. Ma and D. Minda, A unified treatment of some special classes of univalent functions, Proceedings of the Conference on Complex Analysis (Tianjin, 1992), 157-169, Conf. Proc. Lecture Notes Anal., I, Int. Press, Cambridge, MA, 1994.

[47] M. Arif, M. Raza, H. Tang, S. Hussain and H. Khan, Hankel determinant of order three for familiar subsets of analytic functions related with sine functions, Open Mathematics 17 (2019), 1615-1630. https://doi.org/10.1515/math-2019-0132

This is an open access article distributed under the terms of the Creative Commons Attribution License (http://creativecommons.org/licenses/by/4.0/), which permits unrestricted, use, distribution and reproduction in any medium, or format for any purpose, even commercially provided the work is properly cited. 